\title{
Diagnóstico arqueológico de Contagem, Minas Gerais, Brasil
}

\author{
"Archaeological diagnosis of contagem, \\ minas gerais, Brazil
}

\begin{tabular}{l} 
Inês de Oliveira Noronha \\
\hline Paulo Eduardo de Oliveira Enéas \\
\hline Universidade Federal do Rio Grande, Brasil \\
\hline
\end{tabular}

\section{Resumen}

Este artículo hace referencia a una investigación arqueológica en Contagem, ciudad del estado de Minas Gerais, Brasil. El trabajo tiene carácter de diagnóstico para futuras construcciones en la ciudad. En el mismo se presentan nuevas evidencias del potencial arqueológico de la comunidad de Los Arturos, que fueron halladas en el área. El artículo es informativo y es parte de un proyecto investigativo en desarrollo para mantener la herencia cultural de Contagem.

Palabras clave: Contagem, herencia cultural, investigación arqueológica, Los Arturos.

\section{Abstract}

This article makes reference about archaeological study in Contagem, city of Minas Gerais State, Brazil. The work takes diagnostic character for future constructions in town. In this paper presents new evidence ofarchaeological potential of the community of Los Arturos, where was found in the área. This article takes informative character andispart of thea research project for develop ing to maintain the cultural heritage of Contag.

Keywords: Contagem, cultural heritage, arqueologicalsearch, Los Arturos.
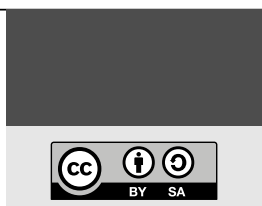

(C) 2019

\section{Correspondencia} de autor:

paolo.deoliveira@usp.br

Recibido:

24/Marzo/2019

Aceptado:

23/Abril/2019

Publicado:

28/Junio/2019 


\section{Introducción}

Este trabajo se realizó con el fin de cumplir con la legislación relativa a la licencia ambiental de un área asignada en la modalidad de lote para acciones constructivas. Se ofrece el diagnóstico de su patrimonio arqueológico, prestándose atención a su registro, investigación, preservación, conservación y divulgación del conocimiento generado. El área en cuestión se encuentra en la Hacienda Vista Alegre, en el barrio de San Sebastián, en el municipio de Contagem, Minas Gerais.

Con esa finalidad se llevó a cabo su estudio para identificar y mapear el potencial arqueológico de las áreas de influencia del proyecto, permitiendo así, los subsidios necesarios y detallar los estudios en las siguientes fases.

Específicamente, en relación con las actividades realizadas, se presenta en este artículo el trabajo arqueológico ejecutado, que se sustentó en la revisión sistemática de la literatura con énfasis en la conformación histórica, etnohistórica de la región, así como en el contexto de la Arqueología Regional. De la misma manera, es relevante para la comprensión de las medidas adoptadas la presentación del marco teórico metodológico en el que se basó el desarrollo de la investigación. Vale señalar el trabajo de campo llevado a cabo por el equipo de arqueología, así como los resultados obtenidos en este esfuerzo. La última parte del artículo se dedica a la descripción y evaluación del impacto en las áreas de interés arqueológico identificadas.

De este modo, se buscó a través del diagnóstico intervencionista evaluar el potencial arqueológico del área e identificar los elementos materiales que portan el patrimonio cultural local. Se partió de la premisa de que el patrimonio arqueológico es herencia cultural de la nación, en la medida en que es el registro físico de la actividad desarrollada de los diferentes actores que participaron en la formación de nuestra sociedad.

Así, su gestión y protección pasan por la realización de estudios que generan nuevos datos y ofrecen a la comunidad elementos para identificar sus raíces culturales y sociales (Morales, 2006). 


\section{Desarrollo}

\section{Conceptos y metodología}

El enfoque teórico asumido en esta investigación fue el de paisaje arqueológico, perspectiva que proporciona el marco necesario para la comprensión de las relaciones que se establecen entre el hombre y el medioambiente, teniendo en cuenta la diversidad cultural, social y material para la elaboración de una historia centrada en el escenario de las actividades humanas del pasado, mostrándose rupturas y continuidades entre el pasado precolonial y la historia posterior (Knapp, \& Ashmore, 1999; Curtoni \& Endere, 2009; Fagundes, 2007).

En todos sus aspectos el trabajo cumplió los requerimientos establecidos por la Ley Federal № 3924/64; en el Artículo 20 de la Constitución Federal de Brasil en 1988 y normativas del SPHAN 07/1988; IPHAN 230/2002 y 28/2003, que prescriben como obligatorio la realización de estudios arqueológicos en los proyectos que tienen el potencial de afectar cualquier objeto cultural. El diagnóstico arqueológico también está regulado por la Resolución de la CONAMA № 001 de 1986, y el Decreto Ley N ${ }^{\circ}$ 25/1937 y el Decreto Ley No. 3551/2000, sobre el patrimonio histórico y artístico y establece el registro de la propiedad inmaterial; se tomó en consideración la Ley № 9605 de 30/03/1998, Sección IV, que se ocupa de los delitos relacionados con el patrimonio cultural.

Con el fin de caracterizar mejor las zonas comprendida por el proyecto, se entiende como Área Directamente Afectada (ADA), al área de la instalación efectiva de la empresa, así como otros sitios en los que realizarian obras de infraestructura que requieren movimiento de tierra, tala y vías de acceso.

El Área de Influencia Directa (AID), se definió teniendo en cuenta el territorio que abarcan las carreteras y vías de acceso al ADA y otras áreas próximas, la microred, hidrográfica local, barrios próximos, etc.

La metodología utilizada en este estudio comprendió los siguientes procedimientos: revisión sistemática de la literatura; entrevistas informales con los residentes en las áreas de impacto; recorridos de campo para el reconocimiento de las ADA y AID; 
realización de perforaciones para verificaciones en el subsuelo y procesamiento de la información diagnóstica obtenida sobre las áreas de estudio.

Con el fin de aumentar los subsidios que permiten la contextualización, descripción e interpretación de los datos sobre el terreno, así como de los bienes patrimoniales identificados en las áreas estudiadas, se ejecutó una revisión sistemática de la literatura. Se realizaron entrevistas informales con los residentes, especialmente con aquellos de mayor edad y con experiencia de vida en la localidad, que se complementaron con recorridos por el ADE y el AID, fueron recorridos sistemáticos con perforaciones para identificar y registrar los diferentes estratos ambientales y los bienes de valor prehistóricos e históricos del lugar.

Los datos recogidos durante el trabajo fueron sistematizados e interpretados atendiendo la necesidad de preparar el informe diagnóstico arqueológico de la zona afectada, el cual se presentó en calidad de un informe final.

\section{Caracterización general del municipio de Contagem}

El municipio de Contagem se halla en la parte central de Minas Gerais, en la región mesometropolitana de Belo Horizonte, más específicamente en la microrregión de Belo Horizonte. Ocupa una superficie de $195,2 \mathrm{~km}^{2}$, y tiene como municipios limítrofes a Esmeraldas, Ribeirão das Neves, Ibirité, Betim y Belo Horizonte. Según el IBGE (2010), posee 600.000 habitantes, por lo que es una de los municipios más poblados de Minas Gerais.

El área de estudio es un terreno que se localiza en un espacio de expansión urbana, a unos $25 \mathrm{~km}$ de la ciudad de Belo Horizonte (Contagem, 2010). En relación con su origen histórico, se estima que la localidad tuvo su proceso de colonización posiblemente a finales del siglo XVII e inicios del XVIII, con la construcción de un puesto de registro que se convirtió en una parada para las tropas de bandeirantes que avanzaban hacia las minas coloniales en busca de oro y de indios. Con los años, fue zona de asentamiento de diversos grupos sociales, que fueron dejando como legado diverso de manifestaciones culturales (Campos, 1991; Costa, 2010). 


\section{Marco histórico}

El proceso de colonización de la zona que llegó a conformar el municipio de Contagem comenzó entre los siglos XVII y XVIII, años en los que los bandeirantes paulistas, especialmente los coordinados por Fernao Dias Paes, en busca de oro y piedras preciosas se adentraban hacia el interior de la colonia recientemente descubierta. Tales expediciones finalmente consolidaron un camino que se fue usado ampliamente, promoviendo la comunicación entre la Capitanía de Sao Paulo y la Sierra del Espinazo, lugar rico en minas de oro (Bonada, 2011).

Más tarde, con la creciente necesidad de contactos con la Capitanía de Río de Janeiro y Bahía, surgieron otras dos rutas, muy transitadas por aventureros, bandeirantes, exploradores, comerciantes, indios, esclavos, conductores de ganado, entre otros. Es de destacar el hecho de que estas tres vías se cruzan en la región conocida como Abóboras, siendo posible encontrar referencias a este cruce en documentos con fecha de 1710.

En relación con el nombre, no se sabe su origen. Lo que se sabe es que no era un lugar urbano y sí una gran área que sirvió como punto de comercialización y distribución de bienes, alimentos y otros bienes que deben formar parte de la zona minera (Bonada, 2011).

Fue en el contexto del continuo flujo de personas que pasaban por el cruce de Abóboras en el que se puede encontrar el origen de Contagem, inicialmente compuesto por pequeños ranchos, casas de conductores de ganado, comerciantes, excavadores, dedicados a la búsqueda de oro en pequeños arroyos. En ese tiempo, el suministro a las zonas mineras era su actividad económica central. Por el intenso flujo de mercancías, personas y capital en la región, la corona portuguesa, instaló un puesto de registro fiscal, para cobrar impuestos. En aquellos años las autoridades de la colonia ejercieron control sobre los territorios ocupados mediante las "oficinas de registro". De hecho, en la comarca Río de las Velhas fueron instalados diez de estas oficinas (Campos y Anastasia, 1991). Estas instalaciones realizaban el control de la circulación de personas y mercancías, cargas y tropas. Como los viajes realizados eran largos, rápidamente estos puntos pasaron a ser lugares de abrigo para pasar la noche. No es de extrañar que el intenso movimiento haya motivado la aparición de campos de cultivos y también la ganadería orientada principalmente a la oferta, la 
supervivencia, además de incrementar los asentamientos existentes (Mercadante, 1973; Morales, 2005).

De este modo, se reitera que el origen de Contagem está particularmente ligado a una de estas oficinas de registro, específicamente la instalada en el año 1716, en las tierras del capitán John de Sousa Souto Maior, en un área conocida como Sitio de Abóbaras, situado en el cruce de los caminos coloniales (Bonada, 2011). Esta era una zona adyacente al terreno de Betim Paes Leme, cuñado de Fernao Dias Paes Leme.

En las inmediaciones del puesto de Abóboras, se consolidó una pequeña ciudad debido al intenso tráfico de personas y mercancías. Inicialmente llamado Arraial de Abóboras y más tarde fue renombrado como Contagem de Abóboras, lo que se debió a que en el lugar, todo lo que por ahí pasaba era contado y gravado con impuestos, por lo que popularmente ganó fuerza el nombre de Contagem (Costa, 2010). Otra importante función que desempeñaba el puesto de registro, fue el cambio del oro en polvo por oro ya pesado, el dueño del oro en polvo lo entregaba a empleados de la Corona y a cambio recibía barras de oro timbradas, con el impuesto (quinto) ya descontado. Estaba estrictamente prohibido I sacar el oro en polvo fuera del área de su extracción, de los caminos y del lugar de intercambio de su polvo por barras de oro, de lo contrario se podía sufrir severos castigos, tales como la deportación a las colonias portuguesas situadas en Asia. Es de destacar que este puesto de control se mantuvo activo hasta 1759. A partir de ese año surgieron nuevas rutas entre los centros mineros, lo que llevó a la instalación de nuevos puestos de control en otros lugares, lo que redujo el flujo de personas y la actividad de los mercados en la región de Contagem (Bonada, 2011).

Se debe mencionar que casi en la misma época se construyó una capilla destinada al culto del santo patrón de los viajeros, San Gonzalo de Amarante. La iniciativa fue responsable de la aparición del nombre "San Gonzalo de Abóbaras", nombre en honor al patrono a la vez que hacía referencia al conteo constante de cabezas de ganado, los esclavos y mercancías gravados en el puesto de registro (Campos \& Anastasia, 1991). A finales del siglo XVIII, y en el siglo XIX, el poblado comenzó a mostrar signos de crecimiento constante, aunque lento.

En gran medida, esto se debió al agotamiento de las regiones mineras, que dieron a la ganadería y a la agricultura un nuevo relieve. Según Bonada (2011), en ese tiempo fue 
cuando llegaron los miembros de las familias tradicionales de la región, tales como Diniz, Macedo, Gonzalves Lina, Silva Costa, Roca, Camargos y Mattos, responsables de la creación de haciendas como Madera, Morro Redondo, Sierra Negra, Abóboras, Riachuleo de las Piedras, pintado, Vista Alegre, Confisco, entre otras.

En relación con su condición administrativa, Contagem fue elevado a la categoría de ciudad en 1911, que comprendía los distritos Camanhá, Veracruz y Vargem Pantana. Esta condición se mantuvo sin cambios hasta 1938 cuando el puesto de conteo perdió su autonomía, convirtiéndose en el distrito de Betim. Así permaneció hasta 1948, año en que retomó la condición de municipio, pero solo con el distrito sede (Fonseca, 1978). Así, se puede afirmar que Contagem presenta un proceso histórico marcado por la diversidad. Pasó de antiguo puesto de registro de las minas coloniales a centro industrial, el territorio siempre fue escenario para diferentes actores sociales, tales como empleados de la Corona portuguesa, comerciantes, campesinos, esclavos y personas anónimas, todos responsables de la memoria histórica y de las tradiciones cultural de la ciudad.

\section{Arqueología regional y patrimonio cultural}

Uno de los primeros estudios reportados en la región tuvo lugar en la región de Lagoa (Laguna) Santa, por el danés Peter Wilhelm Lund. Este investigador descubrió más de 800 cuevas con restos de ocupación humana asociadas con la fauna extinta, lo que contribuyó a reforzar los debates sobre la antigüedad del hombre en esa época (Neves, y Atuí, 2004). La repercusión que se generó, a partir de la década de 1920 en la región comenzó a ser intensamente visitada por los arqueólogos de las misiones extranjeras. En este contexto, se destaca el trabajo del arqueólogo austriaco Jorge Augusto Padberg-Drenkpol, contratado por el Museo Nacional de Río de Janeiro entre los años 1926 y 1929 (Prous, 1992). Posteriormente, entre los años 1935-1960, varias excavaciones se llevaron a cabo en las inmediaciones de Lagoa Santa, realizadas por miembros de la Academia de Ciencias de Minas Gerais. Sin embargo, estas intervenciones carecían de recursos técnicos, por lo que fue imposible el registro detallado de los resultados, así como de la continuidad de los estudios.

Esta realidad solo pudo cambiar a partir de la década de 1970, con el desarrollo del Programa de Investigación Arqueológica en el Valle del San Francisco, en Minas 
Gerais. La iniciativa fue coordinada por el profesor Ondemar Dias Junior, del Instituto Brasileño de Arqueología (IAB), y puede ser entendida como una extensión del Programa Nacional de Investigación Arqueológica (PRONAPA). Como parte de las actividades llevadas a cabo se lograron descubrimientos importantes, tales como los sitios pertenecientes a la Tradición Sapucaí, cerca de la ciudad de Pequi, a unos 100 $\mathrm{km}$ de la ciudad de Contagem. Paralelamente a los trabajos de la IAB, llega a la región, a finales de 1960, la Misión Franco-brasileña, dirigida por Annette Laming-Emperaire y André Prous,

La llegada de investigadores extranjeros fue el marco decisivo para la consolidación del primer Núcleo Científico de Arqueología del Estado. El germen para esta iniciativa fueron las excavaciones sistemáticas llevadas a cabo, tanto en la región metropolitana de Belo Horizonte como en Campo de las Vertientes, iniciadas en 1971 con el apoyo de la Universidad Federal de Minas Gerais (UFMG) y del Instituto del Patrimonio Histórico y Artístico Nacional.

En 1975, André Prous fue contratado por la UFMG, hecho que propició la creación del Sector de Arqueología, concebido como núcleo inicial del futuro Museo del Hombre de Minas Gerais.

En el período comprendido entre las décadas de 1970 y 1990, a mayoría de las investigaciones desarrolladas en la región, fue ejecutada por el Equipo de Arqueología de la UFMG, insertado en el Museo de Historia Natural y en el Jardín Botánico de la UFMG (MHNJB/UFMH). En este sentido, una consulta en la base de datos del IPHAN es suficiente para obtener información que, en este período, fue identificado un número significativo de sitios arqueológicos. A manera de ejemplos, se pueden mencionar aquellos situados en los municipios de Brumadiño, Jequitibá, Papagayos, Baldim, Matoziños, entre otros.

Cabe destacar que a partir de la década de 1990, la actuación del equipo interdisciplinar de la Universidad de Sao Paulo, dirigida por Walter Neves, ganó notoriedad, al realizar un significativo trabajo en la región de Lagoa Santa. Los estudios ejecutados colocaron a la mesoregión metropolitana de Belo Horizonte en el centro de las discusiones internacionales sobre el poblamiento de América (Neves y Piló, 2004). 
En los últimos años, este cuadro de investigaciones fue ampliado, lo que condujo al crecimiento vertiginoso de la arqueología de contrato o preventiva. En buena medida, esto se debe a los estudios llevados a cabo sobre el ámbito empresarial que abarcaban áreas que hasta entonces nunca habían sido abordadas por ningún proyecto de investigación (Monticelli, 2005).

En el caso específico del municipio de Contagem, no fue posible identificar ninguna noticia referente a la existencia de sitios arqueológicos en las bases virtuales del IPHAN. Se sabe, sin embargo, de la presencia de antiguos asentamientos en los municipios limítrofes de lbirité, que consta con dos sitios precoloniales, el sitio Antipoff y el de Serra Boa; y en la ciudad de Belo Horizonte, los sitios Horto Florestal y Córrego do Cardoso, ambos relacionados con la Tradición Aratu-Sapucai.

Con el trabajo de diagnóstico realizado, no fue posible encontrar sitios precoloniales en el área de investigación. Sin embargo, se considera la posibilidad de encontrarlos en la próxima fase del proyecto.

En lo que respecta al patrimonio cultural, este puede ser definido como el conjunto de todos los bienes materiales y/o inmateriales, que, debido al valor expresado, se consideran relevantes en lo que respecta a la identidad y la cultura de un grupo social determinado. Según el IPHAN, no solo incluye a las propiedades aisladas oficiales, sino que en el contexto actual se extiende a la propiedad privada, zonas naturales y hasta el paisaje, incluso, abarca todo un universo de expresiones culturales, así como las antiguas tradiciones de un grupo social para mantenerlas y transmitirlas a las generaciones futuras.

En general, se puede decir que el patrimonio cultural es más que lo material, son también los conocimientos y memorias, maneras de hacer, formas de expresión, celebraciones, fiestas y bailes populares, leyendas, canciones, costumbres; en definitiva, todos los aspectos que se refieren a la identidad de una comunidad determinada.

En el caso particular del municipio de Contagem, al hablar de la herencia cultural conocida, se hace referencia a las expresiones materiales y tradiciones consolidadas desde hace más de 300 años de historia. Preliminarmente es posible destacar algunos bienes de naturaleza material e inmaterial de relevancia histórica y cultural. 
La primera información sobre este tema, puede hallarse en la relación de bienes protegidos de Minas Gerais presentado al Patrimonio ICMS. En ese documento, están listados los siguientes bienes: Capilla de la Inmaculada Concepción y St. Edwirges, la Capilla de Santo Domingo de Guzmán, Casa de Cacos, Casa de la Cultura Nair Mendes Moreira, Cine Teatro Municipal, chimeneas y edificio administrativo de la Compañía de Cemento Portland Itaú, conjunto del Centro Cultural Francisco Firmo de Mattos Filho, conjunto arquitectónico de la calle Dr. Casiano № 102, 130, 140, conjunto urbano; Alcaldía, Capilla de Santa Elena, Centro Deportivo Tancredo Neves, Espacio Popular, Hacienda Vista Alegre, Iglesia Parroquial de Santa María, Parque Gentle Diniz, y finalmente, la Plaza Presidente Tancredo Neves.

Cabe destacar que en el municipio Contagem se encuentra una comunidad de "quilómbolos" (descendientes de esclavos fugitivos) llamada quilombo de Los Arturos. El pueblo está situado en una zona adyacente al proyecto, y mantiene vivo un rico patrimonio intangible, con eventos sociales y de carácter religioso, con celebraciones como la Folia (Hoja) de los Reis, Congado, Candomblé, Batuque, Fiesta de San Sebastián, Juan Mato y la Fiesta de la Abolición.

Vale la pena señalar que el patrimonio cultural de Contagem ha sido divulgado por el Ayuntamiento a través de algunas estrategias. Un buen ejemplo son las acciones promovidas por la Casa de la Cultura Nair Mendes Moreira, donde hoy está el Museo Histórico de Contagem. Se puede mencionar la implementación de proyectos como "Por Dentro de la Historia", "Casa Abierta" y "Panel de Contagito"; al mismo tiempo, acciona consistentemente a favor de la protección de bienes con relevancia histórica y cultural.

\section{Diagnóstico arqueológico}

El diagnóstico del potencial arqueológico de las áreas de influencia, se realizó a partir de expediciones de campo para el reconocimiento de las diferentes áreas ambientales, tomando como guía la realización sistemática de sondeos subterráneos en los puntos más propicios, con el objetivo de detectar cualquier vestigio y/o estructura de valor histórico y cultural. Al mismo tiempo, se realizaba la consulta bibliográfica 
con el fin de obtener la información documental para contextualizar el estudio. Las perforaciones se abrieron con pala de cierre y alcanzaron una profundidad media de $50 \mathrm{~cm}$, teniendo en cuenta el contexto arqueológico local o hasta la desaparición de los indicadores de actividad biológica del suelo, la llamada "tierra negra". Todas las coordenadas de los sitios prospectados se establecieron mediante navegación GPS, referenciados con el DATUM SIRGAS 2000. Al mismo tiempo se evaluaron los perfiles en los barrancos y de los suelos arados, con la finalidad de observar la presencia de cualquier rastro arqueológico.

Se destaca que todas las áreas inspeccionadas fueron sistemáticamente aradas en el pasado. Este grado de antropización reduce la posibilidad de existencia en buenas condiciones de vestigios de la época precolonial, por la alteración sufrida del subsuelo. Sin embargo, al llevar a cabo el trabajo de campo se identificaron tres sitios arqueológicos de valor histórico. Uno de ellos fue Valo de los Camargos, situado en el $A D A_{i}$ los otros dos fueron las ruinas de las haciendas Vista Alegre y la de Las Abóbaras o Bandeiriñas, ubicadas en el IDA. También se documentó la comunidad tradicional de Los Arturos.

\section{Resultados obtenidos}

\section{Sitio Valo de los Camargos}

Esta es una zanja que, supuestamente, en el pasado dividía la hacienda Vista Alegre de la hacienda de las Abóboras. Tiene una profundidad que oscila entre 1,50 m y $2 \mathrm{~m}$ y un ancho entre 0,80 m y $1 \mathrm{~m}$. Actualmente, está cubierta por una densa vegetación y se utiliza en algunos lugares como un espacio para la deposición y quema de basura.

Durante los trabajos de campo la zanja estaba parcialmente cubierta. Fue realizado un sondeo de $50 \mathrm{~cm}$ de profundidad que permitió obtener un sedimento oscuro y con ausencia de cualquier rastro arqueológico. Más importante aún fueron los informes obtenidos con el Sr. Mario Braz da Luz, miembro de la Comunidad quilombos de Los Arturos, quien afirmó que es una zanja abierta por los esclavos, intensamente utilizada por sus familiares en tránsito entre la Hacienda Abóboras y la de Vista Alegre. 


\section{Ruina Hacienda Vista Alegre (AID)}

Son los remanentes de un edificio del siglo XVIII, considerado por la historiografía oficial como una de las primeras construcciones que surgieron en la región de Contagem. En la actualidad, las tierras agrícolas se dividieron en varias parcelas, que fueron vendidas a diferentes propietarios. Han surgido algunos cuestionamientos sobre el hecho de que este edificio sea realmente la antigua sede de Hacienda de Abóboras. Por esto fue llamada Hacienda de las Bandeiriñas.

De esta manera, en relación con los sitios arqueológicos históricos diagnosticados, se sugiere para la próxima fase del trabajo, la continuación de la investigación con la implementación del programa de exploración arqueológica para la limpieza y el análisis del subsuelo mediante cuadrícula, mapeo completo, estudio bibliográfico y documental para elaborar un expediente, permitiendo la inserción cronológica y la comprensión sociocultural del contexto revelado.

\section{La comunidad quilombo de Los Arturos (AID)}

Debido a la importancia de la comunidad para conformar el diagnóstico en el municipio de Contagem, se hará aquí una mejor explicación de la comunidad en cuestión.

Fue posible diagnosticar la presencia de una comunidad de quilombos en el Área de Influencia Directa (AID), conocida como la comunidad de Los Arturos.

Una de las etapas del trabajo fue la contextualización de la zona a través de entrevistas con residentes locales, para ello se utilizaron los datos obtenidos en la investigación para una mejor direccionalidad de las conversaciones con los residentes. Por lo tanto, el equipo de arqueología responsable del trabajo abordó el quilombo de Los Arturos con el objetivo de comprender mejor la historia local, así como tener acceso a fuentes primarias y sus informes sobre la vida cotidiana, costumbres, ritos y otros bienes de importancia extrema para el poblado. 


\section{Contexto}

El quilombo de Los Arturos está situado en el barrio Jardín Vera Cruz, localizado a unos $1.200 \mathrm{~m}$ del Área Directamente Afectada (ADA), donde fue establecido hace más de 120 años y en la que viven unas 80 familias, que suman cerca de 500 personas. De esta cantidad de habitantes, un gran número trabaja en las industrias de la comunidad circundante y en empresas ubicadas en Contagem y Belo Horizonte.

Los residentes de Los Arturos son reconocidos como grandes narradores. Ellos tienen un enorme orgullo por su tradición, religiosidad y unión familiar. Sin embargo, el éxodo de los jóvenes hacia la ciudad y la capital causa desagrado en los ancianos, por romper el ciclo de una vida unida a las tradiciones de la comunidad $y$, sobre todo, la existencia misma de la comunidad. Vale la pena señalar, sin embargo, que el quilombo de Los Arturos es considerado uno de los más originales de Brasil, es un gran e importante patrimonio histórico y cultural de Contagem.

El quilombo de Los Arturos se caracteriza por ser una comunidad quilombo urbana debido a su proximidad a una de las mayores ciudades del estado de Minas Gerais. La comunidad está ubicada a solo $2,5 \mathrm{~km}$ del centro de Contagem, lo que influye en ella la comunidad. A pesar de su sello característico, la zona donde está Los Arturos sigue experimentando un proceso de levantamiento antropológico para su reconocimiento como quilombo urbano. Si hay una opinión positiva después de la finalización de los estudios, el proceso para la titulación del territorio el quilombo de Los Arturos, continuará en el Instituto Nacional de Colonización y Reforma Agraria (INCRA).

\section{Historia}

Los residentes de la comunidad son descendientes de Arthur Camilo Silverio (1880), hijo de Camilo Silverio. Este, natural de Angola, había llegado a Minas Gerais como esclavo en el tercer cuarto del siglo XIX. Por sus años de trabajo el Sr. Silverio en 1888 (año de la abolición de la esclavitud) logró acumular la cantidad de dinero necesario para comprar un lote de tierra de aproximadamente 6,5 hectáreas, en las afueras de la ciudad de Contagem. Es de destacar, que incluso, beneficiado por la ley Vientre Libre, Arthur fue testigo del sufrimiento de sus padres, cometido por los esclavistas. 
Aunque su padre había adquirido tierra, Arthur se vio obligado a trabajar en las haciendas para su supervivencia. En ellas, fue duramente maltratado por sus empleadores, y de hecho a menudo sufría tanto como sus predecesores. Una historia aún no escrita de los descendientes de Arthur Camilo Silverio es lo ocurrido con la muerte de su padre. El Sr. Arthur trató de ver a su hijo, y debido a su actitud, fue golpeado cruelmente en la boca con un instrumento de madera.

Este es un importante hecho en la vida de Arthur, ya que una de las costumbres conservadas por los esclavos africanos y sus descendientes, fue la bendición de sus muertos, con velatorios, rezos y entierro con dignidad. Sin embargo, con el espíritu trabajador y frugal heredado de su padre, logró reunir suficientes recursos para poder establecerse en la tierra dejada por su padre. Arthur Silverio se casó con Carmelinda María da Silva, con quien tuvo 11 hijos, que llegaron a ser los pilares de la comunidad. Entre los herederos está el Sr. Mario Braz da Luz (Tío Mario), localizado y entrevistado por el grupo de esta investigación.

\section{Historia oral}

De importancia para la consolidación de la investigación fue la entrevista con el Sr. Mario, de 78 años, quien realizó actividades como granjero y agente de seguridad y ahora es considerado el "padre de todos" y la máxima autoridad del quilombo de Los Arturos. Es considerado como el "capitán en jefe" de la comunidad y fue reconocido como Mestre Grió por el Ministerio de Cultura. Este señor es visitado por diferentes sectores de la ciudad de Contagem para la celebración de oraciones y bendiciones. Él reside en el lugar hace 70 años, cuando llegó de Esmeraldas junto con su familia.

El Sr. Mario ofreció información sobre cómo era la vida de la comunidad en el pasado. Cuando llegó al lugar las dos haciendas realizaban actividades agrícolas, la Hacienda de las Abóbaras y la de Vista Alegre, que en ese momento pertenecía al Sr. Joviano Camargos. Según el Sr. Mario, las propiedades estaban-divididas por una zanja, que había sido construida en un pasado lejano, por los esclavos. Hizo hincapié en que las dos haciendas fueron empleados miembros de la familia del Sr. Arthuro.

Otro entrevistado, el Sr. Light afirmó haber trabajado para el señor Joviano en pastoreo del ganado, ordeño, siembra de maíz y arroz. En aquel momento, la Hacienda Vista Alegre poseía una casa enorme, con más de 10 habitaciones. La 
casa era tan amplia que, incluso, en ella vivían los empleados. Así, la mayoría vivía en la hacienda realizando diferentes actividades. Los jueves, el Sr. Joviano permitía pescar en el arroyo local, complementando así el sustento. Se supo que la familia de Arthur subsistía de la compra de azúcar y queroseno. En una vida marcada por el trabajo duro, la comunidad se divertía con las dos celebraciones más importantes, que tenían lugar anualmente y que hoy siguen vivas en la tradición local, ellas son la Fiesta de la Abolición y El Congado, en honor a Nuestra Señora del Rosario.

Además de la cultura expresiva y la fuerte religiosidad, la comunidad de Los Arturos realiza, además de las citadas por el Tío Mario, diversas celebraciones de carácter religioso y cultural. Se destaca entre ellas, la Fiesta Joao do Mato, la Folia de los Reyes, los ritos del candomblé (santería) y toques de tambores, realizados en los matrimonios y los bautizos. Vale la pena señalar la existencia del grupo Hijos de Zambi, y la preservación de los conocimientos culinarios.

\section{Celebraciones, expresiones culturales y conocimiento}

La Folia de los Reyes se celebra habitualmente en los meses de diciembre y enero, es la representación de los tres reyes magos que salen a la búsqueda del Niño Dios y se realiza con guitarra, ukelele, acordeón y tambores. El grupo sale en una visita a los hogares comunitarios y donde encuentran un pesebre, realizan cantorías y danzas. Después de toda esta celebración, la comunidad confraterniza con los visitantes en un almuerzo. Los tambores y el candomblé han pasado de generación a generación. Son realizados por los ancianos de la comunidad en los bailes, bodas y bautizos. Durante los tambores, los miembros bailan descalzos, el Candomblé es un rito cerrado e interpretado por los capitanes del grupo. Durante la ceremonia, los capitanes buscan el contacto con Nuestra Señora del Rosario y con los antepasados de la familia Silverio. Esta actividad mantiene una conexión entre los vivos y los antepasados.

La Fiesta de Joao de Mato, en diciembre, es un festival que se celebra cuando se ejecuta la limpia de los cultivos de frijol, maíz y arroz. Según Lucas (2011), esta celebración representa lo ocurrido cuando la zarza invadía las siembras y el personal 
iba a limpiarla; una persona vestida con ropa hecha de hierbas, representa a la zarza a ser eliminada, la gente pasa bailando y cantando con azadas y se encuentra con la "zarza". Se produce un debate entre ella y los "limpiadores", y aquella termina expulsada.

La Fiesta de la Abolición, como lo atestigua el Sr. Mario, es una de las celebraciones más importantes de la comunidad, y tiene lugar en mayo debido a la abolición de la esclavitud en 1888, tiene un carácter cívico y la exaltación a la abolición de la esclavitud, pero también tiene un carácter religioso. Según la profesora Glaura Lucas "es una fiesta basada en la fe que justifica la presencia de los guardias del Congado (manifestación cultural y religiosa afro-brasileira). La experiencia de la vida de los "congaderos" incluye la herencia africana sobre la importancia de los antepasados y de su actuación divina con la Virgen del Rosario a lo largo de la vida de sus descendientes".

La Fiesta de Nuestra Señora del Rosario tiene lugar en octubre, y su fama atrae a muchas personas hacia Contagem. La Congada de Arturo es famosa y atrae a muchos. La festividad tiene como característica principal, la procesión y la danza. Los intérpretes representan al corazón de un rey congoleño y a la devoción hacia Nuestra Señora del Rosario. Se realiza un recorrido por las calles de la comunidad hasta la Iglesia de Nuestra Señora del Rosario, en el municipio de Contagem.

La fiesta dura tres días, pero sus preparativos van más allá de ese período. Como se indica en la página virtual de la Comisión Pro-Indígena de Sao Paulo, es necesario adornar la comunidad y sus calles, la capilla, el territorio, realizar el mantenimiento de la ropa y de los instrumentos, producir los dulces y preparar el almuerzo para más de quinientas personas. Todo esto toma tiempo, lleva una organización comunitaria y necesita de cooperación, lo que demuestra el deseo de la comunidad de perpetuar tal manifestación cultural.

Por último, el compromiso de esta comunidad con el festejo se logra por un sentimiento de amor y devoción por el rito y la historia de la comunidad, así como por su responsabilidad. Es importante tener en cuenta que los habitantes no están obligados a participar en la organización ni en la fiesta; esto ocurre de forma voluntaria, en función con lo sagrado. 
SegúnAredesetal.(2011), losritosdeCongado, porsucarácterreligioso, proporcionanala comunidad experimentar carácter mítico, permite que sus practicantes establezcan una relación con los antepasados y los santos mediante el desarrollo de actuaciones rituales.

Es de destacar la presencia de un grupo creado a principios de 1990 en la comunidad con el fin de preservar los recuerdos y conocimientos en las prácticas musicales y coreografías afro-brasileñas. El grupo Arthuro Filhos de Zambi está formado por jóvenes que quieren aprender y representar las tradiciones de sus predecesores. El reconocimiento que el grupo ha alcanzado ha sido tanto que, rutinariamente, aparecen en actos públicos y privados, además de la colaboración con la Prefectura de Contagem para presentaciones en diversos eventos culturales. Según Aredes et al. (2011), el grupo tiene la importante función de ser una protección de las tradiciones de la comunidad de Los Arturos, pues al corresponderse con diversos ámbitos, terminan por proteger los ritos religiosos existentes en la sociedad actual.

Con respecto al conocimiento de la comunidad, se puede destacar la fabricación del dulce conocido como papa-huevo o amarillito. Este es importante en la comunidad, siendo el objetivo de registros videográficos, con el fin de preservar ese conocimiento. Se ha de tener en cuenta que el color amarillento del dulce se produce en un horno de barro $y$, a diferencia de las bandejas de aluminio, se utilizan hojas de plátano para su cocción, con formas que dependen del artista responsable.

Por lo tanto, es importante tener en cuenta que, además de las influencias externas sufridas por la comunidad por estar insertada en el contexto urbano, la instalación del proyecto afectará directamente la vida cotidiana de Los Arturos. El acceso al proyecto se hará por la avenida adyacente a la comunidad de Los Arturos. Por lo tanto, en una empresa con las dimensiones del proyecto en cuestión, el flujo de personas y coches tendrá un fuerte crecimiento. En esta edición, la economía local se verá afectada, lo que es frecuentemente perjudicial para las comunidades desfavorecidas.

\section{Conlusiones}

Teniendo en cuenta la importancia de la comunidad y su lugar en el área de influencia directa del proyecto, se propuso en el Informe Técnico $N^{\circ} 02 / 2013$, emitido por IPHAN 
la implementación de las siguientes medidas de mitigación: Según los posibles impactos es pertinente un compromiso firmado por el promotor, que incluya una cláusula específica sobre el régimen en el interior del área adjudicada, que muestre de manera explícita el respeto a las prácticas y manifestaciones tradicionales de la comunidad. En las reglas de la comunidad, debe haber un compromiso explícito de los residentes a mantener el control de los cambios de acuerdo con sus deseos en los períodos de la Fiesta de Nuestra Señora del Rosario, cuando se desarrolla La Congada en octubre y la Fiesta de la Abolición en mayo.

\section{Referencias Bibliográficas}

Aredes, R. O. et al. (2011). Hijos de Zambi: Una Nueva Estrategia de Reproducción de Valores y del Conocimiento en la Comunidad Negra de Los Arturos. Belo Horizonte, Brasil: Editorial.

Bonada, M. P. (2011). Contagem: por dentro de la historia. Revista de Educación sobre el Patrimonio. Casa de la Cultura Nair Mendes. Contagem, Brasil: Alcaldía de Contagem

Campos, A. A y Anastasia, C. J. M. (2010). Contagem: Orígenes. Belo Horizonte, Brasil: Ediciones Mazza.

Contagem (Dependencia que expide la ley). (2010, enero 11). Ley Complementaria № 082, por medio de la cual... Contagem, Brasil: Contagem.

Costa, S. C. (2010). La Educación Infantil en la Ciudad de Contagem: Análisis de una Política (1996- 2010) (tesis de maestría, no publicada), Programa de..., Facultad de..., Departamento de..., Universidad Federal de Minas Gerais, Belo Horizonte, Brasil.

Curtoni, R. P. y Endere, M. L. (org.). (2009). Análisis, Interpretación y Gestión en la Arqueología de América del Sur. Buenos Aires, Argentina: Incuapa.

Fagundes, M. (2007). Sistema de asentamiento y tecnología lítica: organización tecnológica y la variabilidad en el registro arqueológico en Xingó, Bajo San Francisco, Brasil (tesis doctoral, no publicada). Londres, Inglaterra (Mae-Usp).

Fonseca, G. (1978). Contagem ante la historia: Edición de la oficina de prensa y relaciones públicas del Gobierno Municipal Contagem. Contagem, Brasil: Gobierno Municipal. 
IPHAN (2012). Términos de referencia para el licenciamiento ambiental, medio ambiente socioeconómico en sus aspectos relacionados con la protección de los bienes de interés cultural. Belo Horizonte, Brasil: IPHAN.

Knapp, A. B. y Ashmore, W. (1999). Paisajes Arqueológicos: Construido, Conceptualizado, Ideacional (pp.1-30). En Ashmore, A.B., Knapp, A.B. (org.). Arqueologías del Paisaje: Las Perspectivas Contemporáneas. Blackwell Publishers.

Lucas, G. (2011). El trabajo de campo en la investigación acción participativa: reflexiones sobre un experimento en curso con la Comunidad Negra de Los Arturos y la Asociación Cultural Heraldos del Gueto en Minas Gerais. Música y Cultura. Florianópolis ¿UFSC?, (6).

Mercadante, P. (1973). Áreas Baldías del Este. Río de Janeiro, Brasil: Zahar.

Monticelli, G. (2005). Arqueología en obras de ingeniería en Brasil: Una crítica a los contextos (tesis doctoral, no publicada), Porto Alegre, Brasil.

Morales, F. (2005). La red urbana de las Minas Coloniales: En las profundidades del tiempo y el espacio (tesis doctoral, no publicada), Sao Paulo, Brasil.

Morales, J. L. (2006). Reflexiones sobre arqueología preventiva. En Mori, V.M. (org.). Patrimonio: Actualizado el debate. Londres, Inglaterra: IPHAN.

Neves, W. A. y Atuí, J. P. V. (2004). El mito de la homogeneidad biológica de la población Paleoindia de Lagoa Santa: Implicaciones Antropológicas. Revista de Antropología, Sao Paulo, (USP), 47(1).

Neves, W. A., Atuí, J. P. V. y Pilo, L. B. (2008). El pueblo de Luzia en busca de los primeros americanos. Sao Paulo, Brasil: Editora Globo.

Prous, A. (1992). Arqueología Brasileña. Brasilia, Brasil: (UNB).

\section{Sitios WWW Consultados}

IBGE (2010). Ciudades: Contagem, Mg. Instituto Brasileño de Geografía y Estadística. Recuperado de: http://www.ibge.gov.br/cidadesat/topwindow.htm 
